\title{
Plant host and drought shape the root associated fungal microbiota in rice
}

\author{
Beatriz Andreo-Jimenez ${ }^{1,2}$, Philippe Vandenkoornhuyse ${ }^{3}$, Amandine Lê Van ${ }^{3}$, Arvid Heutinck ${ }^{1}$, Marie Duhamel ${ }^{3,4}$, \\ Niteen Kadam $^{5}$, S.V. Krishna Jagadish ${ }^{5,6}$, Carolien Ruyter-Spira $^{1}$, Harro Bouwmeester ${ }^{\text {Corresp. }{ }^{1,7}}$ \\ 1 Laboratory of Plant Physiology, Wageningen University, Wageningen, Netherlands \\ 2 Biointeractions \& Plant Health Business Unit, Wageningen University \& Research, Wageningen, Netherlands \\ 3 EcoBio, Université Rennes I, Rennes, France \\ 4 IBL Plant Sciences and Natural Products, Leiden University, Leiden, Netherlands \\ 5 International Rice Research Institute, Los Baños, Philippines \\ 6 Department of Agronomy, Kansas State University, Manhattan, United States \\ 7 Plant Hormone Biology group, Swammerdam Institute for Life Sciences, University of Amsterdam, Amsterdam, Netherlands \\ Corresponding Author: Harro Bouwmeester \\ Email address: h.j.bouwmeester@uva.nl
}

Background and Aim. Water is an increasingly scarce resource while some crops, such as paddy rice, require large amounts of water to maintain grain production. A better understanding of rice drought adaptation and tolerance mechanisms could help to reduce this problem. There is evidence of a possible role of root-associated fungi in drought adaptation. Here, we analyzed the endospheric fungal microbiota composition in rice and its relation to plant genotype and drought.

Methods. Fifteen rice genotypes (Oryza sativa ssp. indica) were grown in the field, under well-watered conditions or exposed to a drought period during flowering. The effect of genotype and treatment on the root fungal microbiota composition was analyzed by $18 \mathrm{~S}$ ribosomal DNA high throughput sequencing. Grain yield was determined after plant maturation.

Results. There was a host genotype effect on the fungal community composition. Drought altered the composition of the root-associated fungal community and increased fungal biodiversity. The majority of OTUs identified belonged to the Pezizomycotina subphylum and 37 of these significantly correlated with a higher plant yield under drought, one of them being assigned to Arthrinium phaeospermum.

Conclusion. This study shows that both plant genotype and drought affect the root-associated fungal community in rice and that some fungi correlate with improved drought tolerance. This work opens new opportunities for basic research on the understanding of how the host affects microbiota recruitment as well as the possible use of specific fungi to improve drought tolerance in rice. 
1 Plant host and drought shape the root associated 2 fungal microbiota in rice

3

4 Beatriz Andreo-Jimenez ${ }^{1, a}$, Philippe Vandenkoornhuyse ${ }^{2}$, Amandine Lê Van $^{2}$, Arvid Heutinck ${ }^{1}$, 5 Marie Duhamel $^{2 b}$, Niteen Kadam ${ }^{3}$, S.V. Krishna Jagadish ${ }^{3, c}$, Carolien Ruyter-Spira ${ }^{1}$, Harro J. 6 Bouwmeester ${ }^{1, \mathrm{~d}}$

7

$8{ }^{1}$ Laboratory of Plant Physiology, Wageningen University, Wageningen, Netherlands

$9{ }^{2}$ EcoBio, Université Rennes I, Rennes, France

${ }^{3}$ International Rice Research Institute, Los Baños, Philippines

11

12

13 a Present address: Biointeractions \& Plant Health Business Unit, Wageningen University \& Research, Wageningen, Netherlands

${ }^{b}$ Present address: IBL Plant Sciences and Natural Products, Leiden University, Leiden, Netherlands

${ }^{c}$ Present address: Department of Agronomy, Kansas State University, Manhattan, United States

d Present address: Plant Hormone Biology group, Swammerdam Institute for Life Sciences, University of Amsterdam, Amsterdam, Netherlands

Corresponding Author:

Harro Bouwmeester ${ }^{1}$

Science Park 904, Amsterdam, 1098 XH, Netherlands

Email address: h.j.bouwmeester@uva.nl

\section{Abstract}

Background and Aim. Water is an increasingly scarce resource while some crops, such as paddy rice, require large amounts of water to maintain grain production. A better understanding of rice drought adaptation and tolerance mechanisms could help to reduce this problem. There is evidence of a possible role of root-associated fungi in drought adaptation. Here, we analyzed the endospheric fungal microbiota composition in rice and its relation to plant genotype and drought. 
31 Methods. Fifteen rice genotypes (Oryza sativa ssp. indica) were grown in the field, under wellwatered conditions or exposed to a drought period during flowering. The effect of genotype and treatment on the root fungal microbiota composition was analyzed by $18 \mathrm{~S}$ ribosomal DNA high throughput sequencing. Grain yield was determined after plant maturation.

Results. There was a host genotype effect on the fungal community composition. Drought altered the composition of the root-associated fungal community and increased fungal biodiversity. The majority of OTUs identified belonged to the Pezizomycotina subphylum and 37 of these significantly correlated with a higher plant yield under drought, one of them being assigned to Arthrinium phaeospermum.

Conclusion. This study shows that both plant genotype and drought affect the root-associated fungal community in rice and that some fungi correlate with improved drought tolerance. This work opens new opportunities for basic research on the understanding of how the host affects microbiota recruitment as well as the possible use of specific fungi to improve drought tolerance in rice.

\section{Introduction}

Climate change is one of the main driving forces affecting the environment. The resulting higher temperatures act to reinforce the effect of drought (Trenberth et al., 2014). Drought periods are one of the main causes of grain yield losses in crops worldwide, especially in drought sensitive crops such as rice (Oryza sativa), the second most produced and consumed crop in the world. To ensure high productivity, rice requires well-watered conditions and almost half of the fresh water used for crop production worldwide is consumed by rice (Barker et al., 2000). As such, improving yield under drought is a major goal in rice breeding.

The root system is in direct contact with the soil, from which the plant absorbs water, and thus root traits are among the critical factors that can potentially ensure good yields under drought stress. Besides the root system and the plant itself, the interaction between plant root and symbiotic microorganisms forming the root microbiota is now considered a major factor in plant performance. These microorganisms may allow the plant to buffer the environmental constraints (Vandenkoornhuyse et al., 2015) and mitigate or suppress soil borne diseases (Kwak et al., 2018). Root colonizers include arbuscular mycorrhizal fungi (Glomeromycota) (Augé, 2001; Smith \& Read, 2008; Singh, 2011), non-mycorrhizal fungal endophytes from the Ascomycota (such as the 
62 Pezizomycotina) and, to a lesser extent, the Basidiomycota. Root-associated fungi have repeatedly 63 been reported to play a role in plant tolerance to stresses (e.g. Selosse, Baudoin \& 64 Vandenkoornhuyse, 2004; Rodriguez et al., 2009). Fungal endophytes have a broad host range and 65 colonize the shoots, roots and rhizomes of their hosts (Rodriguez et al., 2009). They can increase 66 plant biomass (Ernst, Mendgen \& Wirsel, 2003; Redman et al., 2011; Jogawat et al., 2013) and 67 improve tolerance to biotic (Mejía et al., 2008; Maciá-Vicente et al., 2008; Chadha et al., 2015) 68 and abiotic stresses (Hubbard, Germida \& Vujanovic, 2014; Yang, Ma \& Dai, 2014; Azad \& 69 Kaminskyj, 2015).

70 The root fungal microbiota community is not static and changes with environmental factors. 71 Pesticide application, for example, increases the richness of the AM fungal community 72 composition in roots (Vandenkoornhuyse et al., 2003). In contrast, farming practices such as tillage 73 and ploughing are known to decrease species richness of AM fungi in agricultural soils (e.g. 74 Verbruggen \& Kiers, 2010). Monocropping and conventional paddy cultivation also reduce the 75 AMF diversity and colonization in rice and favor the presence of fungal pathogens (Lumini et al., 76 2010; Esmaeili Taheri, Hamel \& Gan, 2016). In traditionally flooded rice fields, root associated 77 fungal species in the Pleosporales and Eurotiales were less abundant than in roots of plants grown 78 in upland fields (Pili et al., 2015).

79 Despite its reported role in plant fitness, the importance of plant colonizing fungal microbiota is 80 underestimated, both in terms of diversity and functionality (Lê Van et al., 2017). Plants cannot be 81 regarded as standalone entities but rather as holobionts comprised of the plant and its associated 82 microbiota where the microbial community provides additional functions to help the cope with 83 environmental changes and stresses (Vandenkoornhuyse et al., 2015). In this conceptual

84 framework, recruitment by the host of micro-organisms when faced with constraints could explain 85 microbiota heterogeneity on the same host in different developmental stage or under changing 86 environmental conditions. If the host indeed exerts control on the recruitment of microorganisms, 87 it is likely that genetic variation for this trait exists. Indeed, the phyllosphere bacterial community 88 in Arabidopsis thaliana (Horton et al., 2014) and wild mustard (Wagner et al., 2016) but also the 89 barley root bacterial microbiota (Bulgarelli et al., 2015) are to some extent host-dependent 90 suggesting that plants indeed exert control on microbial community recruitment from the 91 microorganisms present in the soil. For the present study, we therefore hypothesized that changes 92 that occur within the fungal microbiota community composition when plants experience an 
93 environmental constraint are (partially) determined by the plant genotype. To address this 94 hypothesis, we analyzed the effect of drought on changes in the root associated fungal microbiota 95 of a range of different rice cultivars and whether these changes may play a role in protecting rice 96 against drought.

97

\section{Materials \& Methods}

99

100

101

102

\section{Plant Materials}

Fifteen rice cultivars (Oryza sativa ssp. indica) from the International Rice Research Institute 103 (IRRI, Los Baños, Philippines) were used in our study. Ten out of the 15 cultivars were selected to maximize the genetic variation using the SNP information available from a published study (Zhao et al., 2011). The five additional cultivars were selected based on their drought tolerance phenotype, and their information is available in IRGCIS database: 107 http://www.irgcis.irri.org:81/grc/SearchData.htm (Table S3).

109

110

111

112

113

114

115

116

117

118

119

120

121

122

123

\section{Field site and growing conditions}

All rice plants were grown at IRRI facilities from December 2012 to March 2013. The upland field (used to grow rice under non-flooded conditions) was located at $14^{\circ} 08^{\prime} 50.4^{\prime \prime} \mathrm{N} 121^{\circ} 15^{\prime} 52.1^{\prime \prime E}$. There were 45 field blocks (three per cultivar) $(0.8 \times 2.5$ meters $)$ and each block included 48 plants. The three replicates of each cultivar were analyzed separately. The minimum distance between blocks was three meters. An additional 45 blocks were used for the drought treatment, so in total there were 90 blocks. The soil was a mix of clay (36\%), sand (22\%) and silt (41\%). The plot design was randomized through the field site. Plants were grown in waterlogged conditions until $50 \%$ of the plants reached the flowering stage. Then a drought treatment was imposed on half of the replicates by withholding irrigation. After 12 days of drought, the stressed plots reached $-46 \mathrm{KPa}$ of soil water potential, while the control plot was saturated with water ( $100 \%$ of soil field capacity). There were no rain events during the stress imposition period. Since the plots were maintained under upland conditions with higher sand and silt and during the hotter tropical months of the Philippines, the targeted stress levels were reached in a relatively short duration of 12 days. Then, three soil cores of $10 \times 70 \mathrm{~cm}$ diameter $\times$ length were collected from the center of the plots of the cultivars, pooled together (per block, so giving three replicate samples per genotype) and stored in 
124 plastic bags at $4^{\circ} \mathrm{C}$ until further use. To remove all soil particles, roots isolated from the soil cores 125 were carefully washed with tap water frozen in liquid $\mathrm{N}_{2}$ and stored at $-80^{\circ} \mathrm{C}$ until use.

126

\section{DNA isolation and sequencing}

128 Each root sample was grinded to powder with a mortar and pestle using liquid nitrogen, and DNA 129 was extracted from 60-80 mg of plant material with the DNeasy Plant Mini Kit (Qiagen) following the manufacturers protocol. From the extracted DNA, we amplified a fragment of the 18S SSU rRNA gene using general fungal primers (NS22: 5'-AATTAAGCAGACAAATCACT-3' and SSU0817: 5'-TTAGCATGGAATAATRRAATAGGA-3') (Borneman \& Hartin, 2000) and the following thermocycler conditions during the PCR: $94^{\circ} \mathrm{C}$ for $3 \mathrm{~min} ; 35$ cycles of $94^{\circ} \mathrm{C}$ for $45 \mathrm{~s}$, $59^{\circ} \mathrm{C}$ for $45 \mathrm{~s}\left(-0.1^{\circ} \mathrm{C} /\right.$ cycle $), 72^{\circ} \mathrm{C}$ for $1 \mathrm{~min}$; and $72^{\circ} \mathrm{C}$ for $10 \mathrm{~min}$. Primers were modified to allow the amplicon multiplexing for the sequence production process. Primer modifications and PCR conditions followed Lê Van et al. 2017. To analyze the entire diversity of the fungal community that is associated with roots, including Chytridiomycota, early diverging lineages related to the former Zygomycota (onwards called Zygomycota) and Glomeromycota (Sanders, Clapp \& Wiemken, 1996), SSU rRNA gene primers have been shown to successfully amplify unknown fungal species or groups (Vandenkoornhuyse et al., 2002; Quast et al., 2013; Lê Van et al., 2017). PCR amplicons were purified with AMPure XP beads (Beckman Coulter). Amplicon size was verified with the Agilent High Sensitivity DNA kit (Agilent Technologies), and the concentration measured using the Quant-ITTMPicoGreen ${ }^{2}$ dsDNA Assay kit (Invitrogen). Finally, the purified $560 \mathrm{bp}$ amplicons were all diluted to similar concentration ( $10^{9}$ copies), pooled and sequenced (454 GS FLX+ version Titanium, Roche), following the manufacturer's guidelines. All the PCRs were performed twice and sequenced separately. These true replicates were used within our trimming strategy.

\section{Sequence data trimming and clustering}

After demultiplexing, sequences were filtered to remove reads containing homopolymers longer than 6 nucleotides, undetermined nucleotides, anomalous length and differences (one or more) in the primer. Quality trimming and filtering of amplicons, OTU identification, and taxonomic assignments were carried out with a combination of amplicon data analysis tools and in-house 
155 the filters were clustered using DNAClust (Ghodsi, Liu \& Pop, 2011). Operational Taxonomic 156 Units (OTUs) were generated out of a minimum of two 100\% identical sequences that appeared 157 independently in the different replicates. After these steps, filtering of chimeric sequences was 158 performed using the 'chimeric.uchime' tool within Mothur (v1.31.0, Schloss et al., 2009). The 159 trimming and clustering pipeline used was the same as used in previous studies (e.g. Ben Maamar 160 et al., 2015; Lê Van et al., 2017). The affiliation statistics to identify OTUs were run using the 161 PHYMYCO-DB database (Mahé et al., 2012). A contingency table was produced to perform all 162 the diversity and statistical analyses. Even though the difference in the number of sequences among 163 samples was below $10 \%$, the dataset was rarefied to the same number of sequences using the

164

165 166

167

168

169

170

171

172

173

174

175

176

177

178

179

180

181

182

183

184

185 module VEGAN (Oksanen et al., 2015) in R (R core team, 2013) before statistical analysis. All sequences were uploaded in the European Nucleotide Archive with the accession number PRJEB22764.

\section{The effect of Arthrinium phaeospermum on rice growth}

In order to assess the effect of one of the fungi associated with yield under drought in the present study, the endophytic fungus Arthrinium phaeospermum was used in a pot experiment to study its effect on rice performance. As the original A. phaeospermum strain from the field was not isolated at the time that the experiment was done, eight strains of the species that were available from the CBS-KNAW Fungal Biodiversity Centre (Utrecht, The Netherlands) were tested (Table S4). In total we sowed 144 plants (eight replicates per treatment and fungal strain). As host, the cultivar IR36 (indica rice) was selected, because this cultivar had a higher A. phaeospermum presence in our field experiment. The seed husk was removed and seeds were sterilized with $2 \%$ sodium hypochlorite (v/v) and rinsed several times in sterile distilled water. Seeds were directly sown in small 0.3 liter (L) pots filled with sterilized sand. Plants were watered regularly with modified half-Hoagland nutrient solution and grown during seven days in a climate cell at $28^{\circ} \mathrm{C} / 25^{\circ} \mathrm{C}$ and a $12 \mathrm{~h}$ photoperiod at $75 \%$ relative humidity and a light intensity of $570 \mu$ moles $\mathrm{m}^{-2} \mathrm{~s}^{-1}$. The fungal cultures were grown in Potato Dextrose Agar (PDA) with rifampicin (50 $\mu \mathrm{g} / \mathrm{ml})$. After the fifth day, the upper part of the soil from the pot close to the plant root was inoculated with a $10 \mathrm{~mL}$ diameter agar disc with mycelium, then covered with a bit of soil and grown for another two days when the drought treatment was started, which consisted of water withholding for six days. To avoid that the plants died, they received a fixed amount of water every day (until 50-55\% of field 
186 capacity) to keep the stress high but not to lose all plant available soil water. After the drought 187 period, all plants were collected and fresh and dry weights were quantified. The hyphae 188 colonization was checked under the microscope in some of the samples for a qualitative purpose.

189

\section{Statistical analysis}

191 All the statistical analyses were performed using R (R core team, 2013). From the contingency 192 matrix, OTU richness (number of species), abundance (number of individual OTUs), evenness and 193 diversity index (Shannon H' index) estimators were calculated using the VEGAN (Oksanen et al., 194 2015) and BIODIVERSITYR (Kindt \& Coe, 2005) packages. Statistical differences in these 195 measures were analyzed using ANOVA, with the treatments (control and drought) as factors using 196 the CAR package (Fox \& Weisberg, 2011). To test for a field position effect on the microbial 197 community results, a Mantel Test and correlogram analysis were performed using the VEGAN package. Each root sample was assigned a field position value (based on two coordinates) and the geographical Euclidean distances were calculated. These distances were subsequently compared 200 with the ecological distances (Bray-Curtis method) calculated for the fungal community to analyze

201 if there is a correlation between the field position and the fungal community distance.

202 Fungal community differences between the different treatments were studied using non-metric 203 multidimensional scaling (NMDS) analysis, after removing rare OTUs (OTUs with $<10$ 204 sequences) using the Bray-Curtis statistic to quantify the compositional dissimilarity (Kulczynski, 205 1928). To test whether significant differences exist between fungal communities from control and 206 drought treatments a permutational multivariate analysis of variance (PERMANOVA) was run 207 with the "adonis" function using the NMDS factor scores (VEGAN Package).

208

209 To study the correlation between plant performance and the associated fungal community, a 210 Variation Partitioning analysis (VPA) was performed in VEGAN using the "varpart" function. The 211 VPA model allows to include many factors as variables to study if they can explain the fungal 212 community composition. In the model the OTU relative abundance data (without the rare OTUs) 213 were included as response variable and 'yield' (described by the grain in grams per square meter) 214 and the rice 'host' (described by the Kinship values from the rice genomic map, McCouch et al. 215 2016) as explanatory variables. As a way to calculate the relative response between treatments, the 216 'yield robustness' was calculated by the phenotypic plasticity index (PI) (Valladares, Sanchez- 
217 Gomez \& Zavala, 2006) defined as (yield control - yield drought $_{\text {) }}$ yield control (calculated for each 218 cultivar). This index was included as an explanatory variable together with the 'host' factor in a 219 new VPA model to study how yield robustness under drought is correlated with the community. 220 We also ran a Spearman correlation analysis with the rcorr function in the HMISC package, 221 between the independent OTUs and yield under control and drought treatments; the OTUs 222 positively correlated with plant yield with a $P<0.004$ were selected for further phylogenetic 223 analyzes, as results with P-values below this threshold were not significant (the P-value cutoff was 224 a result of the correction for multiple testing).

225

226

When exploring changes in fungal communities from OTU patterns of plants fungal microbiota exposed to drought conditions, the use qualitative and discrete quantification methods are useful 228 to limit the possibility that changes in community composition (OTUs) be blurred by differences 229 in OTU abundance (Lozupone \& Knight, 2008; Amend, Seifert \& Bruns, 2010; Magurran, 2013). Hence we also estimated the OTU occurrence (presence/absence) in the different treatments for the OTUs positively correlated with yield.

232

To study if yield is linked to phylogenetic relatedness of the root-fungal microbiota, the phylogenetic signal was calculated using the Blomberg's K statistic, which compares the observed signal in a trait to the signal under a Brownian motion model of trait evolution on a phylogeny 236 (Blomberg, Garland \& Ives, 2003) with the PICANTE package (Kembel et al., 2010). The OTU relative abundance matrix was used as a trait, where the mean and standard error was calculated for each OTU. The original Ascomycota tree generated by Maximum Likelihood Estimation was pruned by the yield correlated OTUs. The pruned tree together with the OTUs abundance data was used to calculate the phylogenetic signal.

242 Pruned trees (i.e. where OTUs with less than 10 sequences had been removed) were separately 243 calculated for the main phyla, Ascomycota and Basidiomycota. Sequences were aligned using 244 MAFFT v.7.123b (Katoh \& Standley, 2013) and then trimmed with Gblocks v.0.91b (Castresana, 245 2000). Phylogenetic trees were generated by Maximum Likelihood (ML) using RAxML v.8.00 246 (Stamatakis, 2014), with the General Time Reversible (GTR) model of nucleotide substitution 
248 correlated with yield, a Neighbor Joining (NJ) tree was generated from a pairwise distances matrix 249 of sequences using the SEQINR (Charif \& Lobry, 2007) and APE (Paradis, Claude \& Strimmer, 250 2004) R packages. All trees were edited using iTOL (http://itol.embl.de, (Letunic \& Bork, 2011). 251

252 To analyze the effect of Arthrinium phaeospermum on plant productivity in our pot experiment, a 253 linear model analysis was performed using the STATS package. The response (plant biomass, 254 water content, root to shoot ratio) and the predictors (treatment 'fungus' and treatment 'drought') 255 were included in a fitted linear model that was then used to run an ANOVA analysis.

256 All data and code for the analyses are available as supplementary material.

257

258

259

260

261

262

263

264

265

266

267 268

269

270

271

272

273

274

275

276

277

278

279

\section{Results}

\section{Root - fungal microbiota in rice}

As the samples were selected from a large field experiment, we performed a Mantel Test to check for the presence of field position effects. This analysis showed that there was no strong effect of field position on the fungal community composition for both treatments (Fig. S1). We analyzed a total of 444,757 fungal sequences of $560 \mathrm{bp}$ forming 902 different OTUs (Fig. 1). The sequencing depth was sufficient to describe the root fungal microbiota (Fig. S2). The 18S rRNA marker has been shown to provide adequate species-level resolution for the identification of many fungal groups, with the exception of the Ascomycota (Vandenkoornhuyse et al., 2002). Despite the use of the fungal 18S rRNA gene database PHYMYCO-DB (Mahé et al., 2012) and its better resolution compared to more generalists databases to identify fungal sequences, most of the OTUs did not match to curated sequences of known close relatives (i.e. they are unknown at the species level or higher taxonomic ranking). Among the 902 OTUs detected, only two belonged to the Glomeromycota (i.e. AM fungi). The biggest OTU richness by far was observed for the Ascomycota phylum (784 OTUs), followed by the Basidiomycota (32 OTUs) (Fig. S3). The remaining OTUs belonged to the Chytridiomycota (9 OTUs), Zygomycota (3 OTUs) and an unclassified phylum (72 OTUs). After filtering out the rare OTUs (here defined as OTUs with less than 10 sequences in all analyzed samples), the fungal $\gamma$-diversity measure, $\mathrm{S}$, was 862 and the Shannon diversity index, H', was 3.5. The $\gamma$-diversity in the different treatments was similar, and the majority of OTUs are present under both control and drought (Fig. S4). 
280 The OTU richness and diversity per taxonomic group differ between the control and drought 281 treatment (Fig. 2). The diversity and OTU richness for the main groups (Ascomycota and 282 Basidiomycota) were higher under drought, whereas the unclassified phylum showed the opposite 283 pattern. Using $\alpha$-diversity, there were small differences in fungal microbiota OTU richness under 284 control and drought, both with non-normalized as well as with normalized data: $\mathrm{S}_{\text {control }}=124, \mathrm{~S}$ 285 drought $=132$. An uneven distribution of OTUs in the rice fungal microbiota community structure 286 was observed $(\mathrm{J}$ eveness index $\sim 0.5)$. This observation matches with the Shannon diversity index (H'), which was higher under drought for all the rice cultivars (Fig. 3), due to an increased OTU 288 richness and the presence of less dominant species. This was confirmed by two-way ANOVA analysis $\left(P=9.7 \times 10^{-13} ; \mathrm{F}=71.08 ; \mathrm{Df}=1\right)$. Interestingly, the magnitude of the change in diversity between control and drought was rice cultivar-dependent (Fig. 3) suggesting an effect of the hostplant on fungal biodiversity. Community compositions differed significantly between treatments

292 (Fig. 4). A phylogenetic analysis of all frequent OTUs (without the rare OTUs) was performed for 293 the main phyla: Ascomycota and Basidiomycota (Fig. S4). OTUs within the Sordariomycetes 294 (Pezizomycotina) and an unclassified group (closely related to Sordariomycetes) dominated (Fig. 295 S3).

296

297 To test the statistical significance of host genotype and treatment visualized with the NMDS 298 analysis, a PERMANOVA analysis was performed on the NMDS scores. The NMDS analysis was 299 based on the dissimilarity matrix (Bray-Curtis), but using the rank orders rather than absolute 300 distances for the PERMANOVA gave us less biases linked to data transformation. With both data 301 (Bray-Curtis dissimilarity matrix and NMDS scores) the results were the same. The analysis 302 supports that there is a strong effect of the treatment (control vs. drought) $\left(\mathrm{R}^{2}=0.37 ; P=0.001\right)$ (Fig. 303 4). In conclusion, the data show that rice genotype and drought have a qualitative and quantitative 304 impact on the fungal community associated with the roots.

305

306 Host and treatment effect on root fungal microbiota

307 To further underpin the effect of drought on the fungal community composition we used Variation 308 Partitioning analysis (VPA). This analysis compares the root associated microbial community with 309 factors or a group of factors and tests if any of them is correlated with the microbial community 310 structure. In a first VPA model the factors 'treatment' (control/drought), 'host' (genotype Kinship 
311 values) and 'yield' were included. Both the 'treatment' effect and the combination 'yield' and

312 'treatment' significantly explained the variation in fungal community composition (i.e. response 313 matrix) ( $P=0.001$; coefficient of determination, $\mathrm{R}^{2}$, of 0.22 and 0.38 , respectively) (Fig. S5a). We

314 observed a similar result using the PERMANOVA analysis. The 'host' effect was very small in 315 the VPA analysis $\left(\mathrm{R}^{2}=0.01\right)$, also confirming the PERMANOVA analysis. In a second VPA 316 analysis, we included 'yield robustness' along with the factor 'host' and the abundance of the 317 OTUs for the different treatments (control and drought) and demonstrated a significant 'host' 318 effect on the fungal community under drought $\left(P=0.002\right.$; coefficient of determination $\left.\mathrm{R}^{2}=0.13\right)$ 319 while 'yield robustness' gave no significant effect (Fig S5b). Also 'yield robustness' and OTU 320 abundance under control showed a significant $5 \%$ of explanation by the 'host' $(P=0.05)$ but not by 321 'yield robustness'. Thus fungal community under a stress environment seems to be more relevant 322 for plant yield robustness than when normal conditions.

323

\section{Effect of fungal endophytes on rice fitness}

325 To address the link between the fungal community and plant fitness under drought, each 326 independent OTU was correlated with seed yield (control and drought separately) as a proxy for 327 drought tolerance. We found 37 OTUs that were positively correlated with yield in both treatments $328(\mathrm{R}>0.30 ; P<0.004)$, of which 13 were occurring more under control and 22 more under drought 329 conditions - which therefore are candidates to have a positive effect on drought tolerance - while 330 of two the presence did not change between the treatments (Fig. 5). Thirteen out of the 37 OTUs 331 were assigned to the Pezizomycotina while the other 24 OTUs could not be classified, although 332 they are closely related to the Pezizomycotina sub-phylum.

333

334 Comparing the phylogenetic signal for yield robustness for each OTU in comparison with OTU 335 abundance showed that there was phylogenetic conservation for yield $(\mathrm{K}=6.6, P=0.01)$. This 336 means that phylogenetically related OTUs are more associated with similar yields than random 337 OTUs. This relatedness is solely due to the data under drought $(\mathrm{K}=8.7 ; P=0.03)$.

339 One of the OTUs identified at the species level, Arthrinium phaeospermum, was among the ones 340 contributing significantly to plant yield $(\mathrm{R}=0.08 ; P=0.01)$ and yield robustness $(\mathrm{R}=0.15 ; P=0.01)$ 341 in the VPA analysis. We found other Sordariomycetes (e.g. Chaetomium sp.), Saccharomycetes 
342 and Dothideomycetes that also were associated with increased plant yield under drought.

343 Interestingly, Arthrinium phaeospermum, belongs to the Pezizomycotina subphylum, which is a

344 group that includes the majority of beneficial fungal endophytes, and the species has been

345 described to promote plant growth (Khan et al., 2008). Therefore, we decided to study it in further

346 detail and used a pot experiment to study its effect on rice. Since we did not have access to

347 sufficient field-collected material for isolation of the corresponding field strain, we ordered 6

348 different $A$. phaeospermum strains from CBS and tested their effect on rice growth under control

349 and drought conditions. The A. phaeospermum strains tested did not have a significant positive

350 effect on the plant shoot biomass under control nor drought conditions (Table S1). We did see an

351 interaction between the factors 'fungus' and 'drought' for the majority of variables measured

352 (Table S1). Indeed, the majority of the fungal strains reduced root biomass under drought (Fig. S6)

353 and affected the root to shoot ratio significantly in the case of strains 2, 4, 7 and 8 (Table S2).

354

355

356 Discussion

357

358 Endospheric fungal microbiota detection

359 There is an increased understanding of the complexity of the root fungal microbiota which is not

360

solely limited to Glomeromycota forming AM association, but also includes other fungi belonging

361

362

363

364

365

366

367

368

369

370

371

372 to the Zygomycota, Ascomycota and Basiodiomycota (e.g. Vandenkoornhuyse et al., 2002; Lê Van et al., 2017). In the present study we report for the first time the analysis of the whole fungal microbiome associated with the roots of rice in the field. The largest group of OTUs we detected was the Ascomycota phylum (784 OTUs), followed by the Basidiomycota (32 OTUs) (Fig. S4). The Ascomycota and Basidiomycota are also dominant in the roots of other plant species such as maize (Kuramae et al., 2013), wheat (Vujanovic, Mavragani \& Hamel, 2012), poplar (Shakya et al., 2013) and Agrostis stolonifera (Lê Van et al., 2017), and they are known to include "dark septate endophytes" (DSEs), which are facultative plant symbionts (Rodriguez et al., 2009).

In this study, the diversity values $\left(\mathrm{H}^{\prime}=3,5 ; \mathrm{S}=862\right)$ are of the same order of magnitude as in other crops. We found a lower H' and different community structure than in chickpea for which a H' of about 4.7 and $\mathrm{S}$ of about 800 have been reported (Bazghaleh et al., 2015) but a higher H' and S than in arctic plants for which an H' of 2.8 and $\mathrm{S}$ of 60 have been reported (Zhang \& Yao, 2015). 
373 For other monocots such as wheat: H' 1.8; S 18, and maize: H' 0.9; S 9 (Bokati, Herrera \&

374 Poudel, 2016) the values are also quite a bit lower than our values, although for the latter the fungal

375 community analysis was done in a very different way. Thus, the rice genotypes used in the present

376 study appeared to recruit a rather high number of fungal species. Possibly, host defense was

377 lowered due to stress and/or plants signaled for help, which resulted in additional fungal species

378 to colonize the roots. The high OTU richness found in the rice root fungal endosphere when

379 compared with other studies, could also be an effect of the primer choice or could be related to the

380 fact that rice is growing in a very different and specific environment in comparison to the other

381 plant species (i.e. in the tropics in a water saturated agroecosystem).

382

\section{Drought affects the endophytic fungal microbiota}

384 It has been reported that the soil fungal community composition changes under drought resulting 385 in a decreased $\alpha$-diversity (Hawkes et al., 2011; Cregger et al., 2012; Seema B. Sharma \& 386 Thivakaran A. Gobi, 2016; Zhang et al., 2016). As far as we know, the consequence of drought on 387 the root associated fungal microbiota has not been investigated before under field conditions. In 388 the present study we clearly demonstrate that the rice endospheric fungal microbiota composition 389 changes under drought stress (Fig. 4) and results in an increased richness of fungal OTUs within 390 rice-roots for all the 15 rice cultivars tested (Fig. 3). Increased fungal richness could be interpreted 391 as an active recruitment of additional fungi by the rice root to face the environmental stress 392 although we cannot exclude that this is the result of the reverse process: fungi actively colonize 393 the root compartment to escape from the drought effect. Nevertheless, a higher fungal diversity 394 could represent a better pool for subordinate species (less abundant ones), which may have a large 395 influence on certain ecosystems and can potentially improve plant productivity under drought 396 conditions (Mariotte et al., 2015). The increase in fungal species richness may result in the 397 enrichment in additional functions enabling to mitigate the consequences of drought on the host 398 plant. Also other studies suggest that fungi have an important effect on plant fitness under drought 399 conditions (Lau \& Lennon, 2012; Kaisermann et al., 2015; Classen et al., 2015). In sorghum it has 400 been shown that when water levels are extreme (drought or flooding), roots are colonized by fewer

401 AM fungal species, however at the same time the abundance of these species increases probably 402 because they are more adapted to the new conditions. In those experiments, plant biomass was not 
403 affected by the water regime, but phosphate uptake was increased as a result of a change in the 404 root colonization of plants under non-flooded conditions (Deepika \& Kothamasi, 2015).

405 Glomeromycota species richness and abundance increased under drought within a diverse panel 406 of plants including wild and cultivated species (Tchabi et al., 2008). Strikingly, in the present 407 study, we only observed two OTUs representing Glomeromycota within the fungal microbial 408 community and they were not affected by drought. Although we know that the fungal microbiota 409 is not only composed of Glomeromycota (e.g. (Vandenkoornhuyse et al., 2002), in our experiment 410 rice is unexpectedly poor in AM fungal colonizers in comparison to other Poaceae. For example, 411 in a study on Agrostis stolonifera and using the same methodological approach as in the present 412 study, the Glomeramycota represented $10 \%$ of the root fungal microbiota (Lê Van et al., 2017). 413 As already commented in the introduction, monocropping and conventional paddy cultivation have 414 been shown to reduce the AMF diversity and colonization in rice, which likely explains the low 415 Glomeramycota representation in the present study.

416 The majority of the OTUs that increased in frequency under drought in our study belong to the 417 Pezizomycotina subphylum, the most abundant subphylum in the Class II fungal endophytes 418 (Rodriguez et al., 2009). They are well-known for their role in plant performance, boosting plant 419 growth and buffering the effect of environmental stresses and protecting their host against 420 pathogens (Maciá-Vicente et al., 2009; Jogawat et al., 2013; Azad \& Kaminskyj, 2015). If looking 421 at other individual OTUs there are changes in their abundance between treatments and/or rice 422 cultivars, however those changes are not following a pattern as a taxonomic group or the 423 description we get at species level is not enough to make further conclusions.

424

\section{Host genotype affects the fungal microbiome response to drought}

426 Using VPA we showed that the host genotype affects the structure of the root associated fungal 427 community, also in response to drought ('host' effect: $\mathrm{R}^{2}=0.13 ; P=0.01$ ) (Fig. S5). Previous studies 428 using Arabidopsis thaliana and barley also show a host-genotype effect on the root associated 429 microbiome (Lundberg et al., 2012; Bulgarelli et al., 2015), However, in maize and Microthlaspi 430 spp. the root endophyte community composition did not seem to depend on the host genotype, but 431 was largely determined by the geographical distribution where these cultivars are coming from 432 (Peiffer et al., 2013; Glynou et al., 2016). Using a GWAS approach for the phyllosphere 433 microbiome composition of Arabidopsis thaliana, it was shown that the fungal and bacterial 
434 community on leaves is determined at least in part by plant loci, in this case by loci responsible 435 for defense and cell wall integrity (Horton et al., 2014). Recently, a new study has shown that 436 drought induces changes in the root bacterial and fungal endophytic community in four rice 437 cultivars under greenhouse conditions (Santos-Medellín et al., 2017), supporting what we observe 438 in our study in the field.

439 The results of the present study clearly show that changes occur within the fungal microbiota 440 community composition when plants experience an environmental constraint (Fig. 4). The 441 increased root fungal endophytic diversity could be the result of migration of soil fungi to the roots 442 to survive the drought conditions. However, the significant genotype effect on the fungal 443 community structure under drought (Fig. S5), strongly suggests that active recruitment by the plant 444 host of fungal species (also) occurs. Potentially, this enrichment of plant-microbiota can buffer the 445 effects of the drought stress (Vandenkoornhuyse et al., 2015). A host-plant preference has also 446 been shown in studies analyzing AM fungal communities (Martínez-García \& Pugnaire, 2011; 447 Torrecillas, Alguacil \& Roldán, 2012) even among co-occurring plant species within the Poaceae 448 (Vandenkoornhuyse et al., 2003). This observation was later explained by the ability of plants to 449 filter the colonizer by a carbon embargo toward less beneficial AM fungi (Kiers et al., 2011; 450 Duhamel \& Vandenkoornhuyse, 2013). We are currently further exploring the role of the rice 451 plant-host in the recruitment of root-associated fungal microbiota using plant genetics approaches. 452

453 Root fungal microbiota and rice grain yield

454 OTUs that are closely related to each other showed similar correlation values with rice grain yield 455 as there is a strong phylogenetic signal between all yield correlated OTUs $(\mathrm{K}=6.6 ; P=0.01)$. Intriguingly, these OTUs are more abundant under drought (Fig. 5), suggesting that they may play a role in the tolerance of rice to drought. In an earlier study, inoculation of rice with fungal Type II endophytes such as Fusarium culmorum and Curvularia protuberata resulted in a higher growth rate and yield and a reduced water consumption. Moreover, the rice plants grown under drought stress were more intensively colonized by these fungi in comparison to control plants (Redman et al., 2011). In the present study we identified 37 different OTUs that belong to the Pezizomycotina which all positively correlated with yield in plants that were exposed to drought (Fig. 5). This might be due to one particular fungal OTU or alternatively might be the consequence of a complex 464 synergistic effect of different OTUs. 
465

466 Among these fungi there was Arthrinium phaeospermum. Arthrinium species are often associated 467 with plants from the Poaceae family, suggesting a certain level of host specificity (Yuan et al., 468 2011). To confirm the role of $A$. phaeospermum, different strains of this species were used in a pot 469 experiment. Under control conditions no significant effect of the inoculation was observed on plant 470 shoot biomass, while root biomass was decreased by some of the strains under drought (Table S2). 471 Root biomass investment (root to shoot ratio) under drought was lower for plants inoculated with 472 some of the strains (Table S2; $P<0.05$ ). These results are counter-intuitive because in the 473 community analysis, A. phaeospermum was correlated with yield, especially under drought as 474 shown by the VPA analysis. The most likely explanation for this is that we did not use the $A$. 475 phaeospermum strain that caused the effect in the field because we used publicly available strains. 476 Also, in the pot experiment biomass was analyzed instead of yield. Another possible explanation 477 is that the OTU we described as A. phaeospermum is actually a different, though closely related, 478 species. To further examine this discrepancy, it will be necessary to isolate the corresponding strain 479 from the field and/or plant material. Another possible explanation is that the yield effect is not 480 directly due to A. phaeospermum but to other microorganism(s) that were not analysed in our study 481 (e.g. bacteria) that are correlated with the presence of $A$. phaeospermum. Drought tolerance may 482 be the result of a synergistic/antagonistic effect between $A$. phaeospermum and these other 483 microorganisms (Larimer, Bever \& Clay, 2010; Aguilar-Trigueros \& Rillig, 2016), while we 484 studied the effect of a single fungal isolate. Likewise, a perturbation of the root microbial 485 community induced by the inoculation may have blurred any positive effects.

486 A higher root:shoot ratio and a longer root length are often characteristics for rice cultivars that 487 are more drought tolerant, as they are good indicators for a higher water uptake capacity (Comas 488 et al., 2013; Paez-Garcia, 2015). We did not record the root length in the pot experiment, so it 489 could be that some of the fungal strains may have had an impact on root length rather than on root 490 biomass. Furthermore, the effect of drought on the root to shoot ratio depends on the plant growth 491 stage, which is most evident in older plants (Silva, Kane \& Beeson, 2012). Therefore, in the 492 relatively young plants that were used in the present study we may have missed the effect that the 493 fungi may have on root architectural changes in older plants. These possibilities should be 494 considered for future studies with the same research questions. 
496 Conclusions

497

498 Our study illustrates that the root associated fungal community in rice changes under drought, 499 resulting in a higher species diversity in the rice-root endosphere. It also shows the presence of 500 specific OTUs (belonging to the Pezizomycotina) is correlated with yield, and the relative 501 abundance of these OTUs increases under drought. Finally, we also show that, under drought, the 502 rice genotype has a significant effect on the fungal community composition.

503 Roots are interesting to search for beneficial-plant growth promoting fungi (Fonseca-García et al., 504 2016; Angel et al., 2016). With sufficient knowledge, we can potentially compose 'functional OTU 505 clusters', specifically tailored for a crop plant species, that we know may have a positive impact 506 on plant performance. This microbial consortium could then be applied in the field to boost plant 507 productivity under periods of stress. However, only a maximum of $1.0 \%$ of soil microorganisms 508 can be cultured under standard conditions. Thus, studying the roles of microbiota in biological and 509 ecological soil processes remains a challenge (Rehman, Sayeed, Mohd Akhtar \& Siti Nor Akmar 510 Abdullah, 2016), specially for possible application in agriculture. Nonetheless, metagenomics and 511 metabarcoding studies can yield valuable information that could help us to exploit microbial 512 communities and further investigate how microbial 'clusters' are working together to improve 513 plant fitness under stressful environments.

514

515

516

517 We thank support staff of IRRI for their help with sample collection and processing and the Human 518

519

520

521

522

523

524

525

526

527

\section{Acknowledgements}

and Environmental Genomics platform (https://geh.univ-rennes1.fr/) and S. Michon-Coudouel for technical support in the library preparation and sequencing, and J.G. Maciá-Vicente for providing $\mathrm{R}$ scripts for some of the statistical analyzes and his support with some of the phylogenetic analyzes.

\section{References}

Aguilar-Trigueros CA., Rillig MC. 2016. Effect of different root endophytic fungi on plant community structure in experimental microcosms. Ecology and Evolution 6:8149-8158. DOI: $10.1002 /$ ece 3.2416. 
528 Amend AS., Seifert KA., Bruns TD. 2010. Quantifying microbial communities with 454

529

530

531

532

533

534

535

536

537

538

539

540

541

542

543

544

545

546

547

548

549

550

551

552

553

554

pyrosequencing: does read abundance count? Molecular Ecology 19:5555-5565. DOI: 10.1111/j.1365-294X.2010.04898.x.

Angel R., Conrad R., Dvorsky M., Kopecky M., Kotilínek M., Hiiesalu I., Schweingruber F., Doležal J. 2016. The Root-Associated Microbial Community of the World's Highest Growing Vascular Plants. Microbial Ecology 72:394-406. DOI: 10.1007/s00248-0160779-8.

Augé RM. 2001. Water relations, drought and vesicular-arbuscular mycorrhizal symbiosis. Mycorrhiza 11:3-42.

Azad K., Kaminskyj S. 2015. A fungal endophyte strategy for mitigating the effect of salt and drought stress on plant growth. Symbiosis:1-6. DOI: 10.1007/s13199-015-0370-y.

Barker R., Dawe D., Tuong TP., Bhuiyan SI., Guerra LC. 2000. The outlook for water resources in the year 2020: challenges for research on water management in rice production. International Rice Commission Newsletter 49:7-21.

Bazghaleh N., Hamel C., Gan Y., Tar'an B., Knight JD. 2015. Genotype-Specific Variation in the Structure of Root Fungal Communities Is Related to Chickpea Plant Productivity. Applied and Environmental Microbiology 81:2368-2377. DOI: 10.1128/AEM.03692-14.

Ben Maamar S., Aquilina L., Quaiser A., Pauwels H., Michon-Coudouel S., Vergnaud-Ayraud V., Labasque T., Roques C., Abbott BW., Dufresne A. 2015. Groundwater isolation governs chemistry and microbial community structure along hydrologic flowpaths. Frontiers in Microbiology 6. DOI: 10.3389/fmicb.2015.01457.

Blomberg SP., Garland T., Ives AR. 2003. Testing for Phylogenetic Signal in Comparative Data: Behavioral Traits Are More Labile. Evolution 57:717-745. DOI: 10.1111/j.00143820.2003.tb00285.x.

Bokati D., Herrera J., Poudel R. 2016. Soil Influences Colonization of Root-Associated Fungal Endophyte Communities of Maize, Wheat, and Their Progenitors. Journal of Mycology 2016:e8062073. DOI: 10.1155/2016/8062073.

Peer) reviewing PDF | (2018:11:32645:1:1:NEW 26 May 2019) 
555 Borneman J., Hartin RJ. 2000. PCR Primers That Amplify Fungal rRNA Genes from 556 Environmental Samples. Applied and Environmental Microbiology 66:4356-4360.

557 Bulgarelli D., Garrido-Oter R., Münch PC., Weiman A., Dröge J., Pan Y., McHardy AC., Schulze558 Lefert P. 2015. Structure and Function of the Bacterial Root Microbiota in Wild and

559

560

561

562

563

564

565

566

567

568

569

570

571

572

573

574

575

576

577

578

579

580

581 Domesticated Barley. Cell Host \& Microbe 17:392-403. DOI: 10.1016/j.chom.2015.01.011.

Castresana J. 2000. Selection of Conserved Blocks from Multiple Alignments for Their Use in Phylogenetic Analysis. Molecular Biology and Evolution 17:540-552. DOI: 10.1093/oxfordjournals.molbev.a026334.

Chadha N., Mishra M., Rajpal K., Bajaj R., Choudhary DK., Varma A. 2015. An ecological role of fungal endophytes to ameliorate plants under biotic stress. Archives of Microbiology 197:869-881. DOI: 10.1007/s00203-015-1130-3.

Charif D., Lobry JR. 2007. SeqinR 1.0-2: A Contributed Package to the R Project for Statistical Computing Devoted to Biological Sequences Retrieval and Analysis. In: Bastolla DU, Porto PDM, Roman DHE, Vendruscolo DM eds. Structural Approaches to Sequence Evolution. Biological and Medical Physics, Biomedical Engineering. Springer Berlin Heidelberg, 207-232. DOI: 10.1007/978-3-540-35306-5_10.

Classen AT., Sundqvist MK., Henning JA., Newman GS., Moore JAM., Cregger MA., Moorhead LC., Patterson CM. 2015. Direct and indirect effects of climate change on soil microbial and soil microbial-plant interactions: What lies ahead? Ecosphere 6:1-21. DOI: 10.1890/ES15-00217.1.

Comas LH., Becker SR., Cruz VMV., Byrne PF., Dierig DA. 2013. Root traits contributing to plant productivity under drought. Frontiers in Plant Science 4. DOI: 10.3389/fpls.2013.00442.

Cregger MA., Schadt CW., McDowell NG., Pockman WT., Classen AT. 2012. Response of the Soil Microbial Community to Changes in Precipitation in a Semiarid Ecosystem. Applied and Environmental Microbiology 78:8587-8594. DOI: 10.1128/AEM.02050-12. 
582 Deepika S., Kothamasi D. 2015. Soil moisture-a regulator of arbuscular mycorrhizal fungal 583 community assembly and symbiotic phosphorus uptake. Mycorrhiza 25:67-75. DOI:

584

585

586

587

588

589

590

591

592

593

594

595

596

597

598

599

600

601

602

603

604

605

606

607

608 10.1007/s00572-014-0596-1.

Duhamel M., Vandenkoornhuyse P. 2013. Sustainable agriculture: possible trajectories from mutualistic symbiosis and plant neodomestication. Trends in Plant Science 18:597-600. DOI: $10.1016 /$ j.tplants.2013.08.010.

Ernst M., Mendgen KW., Wirsel SGR. 2003. Endophytic Fungal Mutualists: Seed-Borne Stagonospora Spp. Enhance Reed Biomass Production in Axenic Microcosms. Molecular Plant-Microbe Interactions 16:580-587. DOI: 10.1094/MPMI.2003.16.7.580.

Esmaeili Taheri A., Hamel C., Gan Y. 2016. Cropping practices impact fungal endophytes and pathogens in durum wheat roots. Applied Soil Ecology 100:104-111. DOI: 10.1016/j.apsoil.2015.12.007.

Fonseca-García C., Coleman-Derr D., Garrido E., Visel A., Tringe SG., Partida-Martínez LP. 2016. The Cacti Microbiome: Interplay between Habitat-Filtering and Host-Specificity. Plant Biotic Interactions:150. DOI: 10.3389/fmicb.2016.00150.

Fox J., Weisberg S. 2011. An R Companion to Applied Regression. SAGE.

Ghodsi M., Liu B., Pop M. 2011. DNACLUST: accurate and efficient clustering of phylogenetic marker genes. BMC Bioinformatics 12:271. DOI: 10.1186/1471-2105-12-271.

Glynou K., Ali T., Buch A-K., Haghi Kia S., Ploch S., Xia X., Çelik A., Thines M., Maciá-Vicente JG. 2016. The local environment determines the assembly of root endophytic fungi at a continental scale: Continental-scale distribution of root endophytes. Environmental Microbiology 18:2418-2434. DOI: 10.1111/1462-2920.13112.

Hawkes CV., Kivlin SN., Rocca JD., Huguet V., Thomsen MA., Suttle KB. 2011. Fungal community responses to precipitation. Global Change Biology 17:1637-1645. DOI: 10.1111/j.1365-2486.2010.02327.x.

Horton MW., Bodenhausen N., Beilsmith K., Meng D., Muegge BD., Subramanian S., Vetter MM., Vilhjálmsson BJ., Nordborg M., Gordon JI., Bergelson J. 2014. Genome-wide 
609

610

611

612

613

614

615

616

617

618

619

620

621

622

623

624

625

626

627

628

629

630

631

632

633

634

635

association study of Arabidopsis thaliana leaf microbial community. Nature Communications 5. DOI: $10.1038 /$ ncomms6320.

Hubbard M., Germida J j., Vujanovic V. 2014. Fungal endophytes enhance wheat heat and drought tolerance in terms of grain yield and second-generation seed viability. Journal of Applied Microbiology 116:109-122. DOI: 10.1111/jam.12311.

Jogawat A., Saha S., Bakshi M., Dayaman V., Kumar M., Dua M., Varma A., Oelmüller R., Tuteja N., Johri AK. 2013. Piriformospora indica rescues growth diminution of rice seedlings during high salt stress. Plant Signaling \& Behavior 8. DOI: 10.4161/psb.26891.

Kaisermann A., Maron PA., Beaumelle L., Lata JC. 2015. Fungal communities are more sensitive indicators to non-extreme soil moisture variations than bacterial communities. Applied Soil Ecology 86:158-164. DOI: 10.1016/j.apsoil.2014.10.009.

Katoh K., Standley DM. 2013. MAFFT multiple sequence alignment software version 7: improvements in performance and usability. Molecular Biology and Evolution 30:772780. DOI: $10.1093 / \mathrm{molbev} / \mathrm{mst} 010$.

Kembel SW., Cowan PD., Helmus MR., Cornwell WK., Morlon H., Ackerly DD., Blomberg SP., Webb CO. 2010. Picante: R tools for integrating phylogenies and ecology. Bioinformatics 26:1463-1464. DOI: 10.1093/bioinformatics/btq166.

Khan SA., Hamayun M., Kim H., Yoon H., Seo J., Choo Y., Lee I., Kim S., Rhee I., Kim J. 2008. A new strain of Arthrinium phaeospermum isolated from Carex kobomugi Ohwi is capable of gibberellin production. Biotechnology Letters 31:283-287. DOI: 10.1007/s10529-0089862-7.

Kiers ET., Duhamel M., Beesetty Y., Mensah JA., Franken O., Verbruggen E., Fellbaum CR., Kowalchuk GA., Hart MM., Bago A., Palmer TM., West SA., Vandenkoornhuyse P., Jansa J., Bucking H. 2011. Reciprocal rewards stabilize cooperation in the mycorrhizal symbiosis. Science 333:880-2. DOI: 10.1126/science.1208473.

Kindt R., Coe R. 2005. Tree diversity analysis: A manual and software for common statistical methods for ecological and biodiversity studies. World Agroforestry Centre (ICRAF). 
636 Kulczynski S. 1928. Die Pflanzenassoziationen der Pieninen. Suppl. II (1927):57-203.

637 Kuramae EE., Verbruggen E., Hillekens R., de Hollander M., Röling WFM., van der Heijden

638 MGA., Kowalchuk GA. 2013. Tracking Fungal Community Responses to Maize Plants by

639 DNA- and RNA-Based Pyrosequencing. PLoS ONE 8:e69973. DOI:

$640 \quad$ 10.1371/journal.pone.0069973.

641 Kwak M-J., Kong HG., Choi K., Kwon S-K., Song JY., Lee J., Lee PA., Choi SY., Seo M., Lee 642 HJ., Jung EJ., Park H., Roy N., Kim H., Lee MM., Rubin EM., Lee S-W., Kim JF. 2018.

643 Rhizosphere microbiome structure alters to enable wilt resistance in tomato. Nature 644 Biotechnology. DOI: 10.1038/nbt.4232.

645 Larimer AL., Bever JD., Clay K. 2010. The interactive effects of plant microbial symbionts: a 646 review and meta-analysis. Symbiosis 51:139-148. DOI: 10.1007/s13199-010-0083-1.

647 Lau JA., Lennon JT. 2012. Rapid responses of soil microorganisms improve plant fitness in novel 648 environments. Proceedings of the National Academy of Sciences 109:14058-14062. DOI: $649 \quad 10.1073 /$ pnas.1202319109.

650 Lê Van AL., Quaiser A., Duhamel M., Michon-Coudouel S., Dufresne A., Vandenkoornhuyse P. 651 2017. Ecophylogeny of the endospheric root fungal microbiome of co-occurring Agrostis 652 stolonifera. PeerJ 5:e3454. DOI: 10.7717/peerj.3454.

653 Letunic I., Bork P. 2011. Interactive Tree Of Life v2: online annotation and display of phylogenetic 654 trees made easy. Nucleic Acids Research 39:W475-W478. DOI: 10.1093/nar/gkr201.

655 Lozupone CA., Knight R. 2008. Species Divergence and the Measurement of Microbial Diversity. 656 FEMS microbiology reviews 32:557-578. DOI: 10.1111/j.1574-6976.2008.00111.x.

657 Lumini E., Vallino M., Alguacil MM., Romani M., Bianciotto V. 2010. Different farming and 658 water regimes in Italian rice fields affect arbuscular mycorrhizal fungal soil communities. 659 Ecological Applications 21:1696-1707. DOI: 10.1890/10-1542.1.

660 Lundberg DS., Lebeis SL., Paredes SH., Yourstone S., Gehring J., Malfatti S., Tremblay J., 661 Engelbrektson A., Kunin V., Rio TG del., Edgar RC., Eickhorst T., Ley RE., Hugenholtz 
662

663

664

665

666

667

668

669

670

671

672

673

674

675

676

677

678

679

680

681

682

683

684

685

686

687

688

P., Tringe SG., Dangl JL. 2012. Defining the core Arabidopsis thaliana root microbiome. Nature 488:nature11237. DOI: 10.1038/nature11237.

Maciá-Vicente JG., Jansson H-B., Mendgen K., Lopez-Llorca LV. 2008. Colonization of barley roots by endophytic fungi and their reduction of take-all caused by Gaeumannomyces graminis var. tritici. Canadian Journal of Microbiology 54:600-609. DOI: 10.1139/W08047.

Maciá-Vicente J g., Rosso L c., Ciancio A., Jansson H-B., Lopez-Llorca L v. 2009. Colonisation of barley roots by endophytic Fusarium equiseti and Pochonia chlamydosporia: Effects on plant growth and disease. Annals of Applied Biology 155:391-401. DOI: 10.1111/j.17447348.2009.00352.x.

Magurran AE. 2013. Measuring Biological Diversity. John Wiley \& Sons.

Mahé S., Duhamel M., Le Calvez T., Guillot L., Sarbu L., Bretaudeau A., Collin O., Dufresne A., Kiers ET., Vandenkoornhuyse P. 2012. PHYMYCO-DB: A Curated Database for Analyses of Fungal Diversity and Evolution. PLoS ONE 7:e43117. DOI: 10.1371/journal.pone.0043117.

Mariotte P., Robroek BJM., Jassey VEJ., Buttler A. 2015. Subordinate plants mitigate drought effects on soil ecosystem processes by stimulating fungi. Functional Ecology 29:15781586. DOI: $10.1111 / 1365-2435.12467$.

Martínez-García LB., Pugnaire FI. 2011. Arbuscular mycorrhizal fungi host preference and site effects in two plant species in a semiarid environment. Applied Soil Ecology 48:313-317. DOI: 10.1016/j.apsoil.2011.04.003.

Mejía LC., Rojas EI., Maynard Z., Bael SV., Arnold AE., Hebbar P., Samuels GJ., Robbins N., Herre EA. 2008. Endophytic fungi as biocontrol agents of Theobroma cacao pathogens. Biological Control 46:4-14. DOI: 10.1016/j.biocontrol.2008.01.012.

Oksanen J., Blanchet FG., Kindt R., Legendre P., Minchin PR., O’Hara RB., Simpson GL., Solymos P., Stevens MHL., Wagner H. 2015. Package 'vegan': community ecology package, version 2.3-2. 
689 Paez-Garcia 2015. Root Traits and Phenotyping Strategies for Plant Improvement.

690 Paradis E., Claude J., Strimmer K. 2004. APE: Analyses of Phylogenetics and Evolution in R 691 language. Bioinformatics 20:289-290. DOI: 10.1093/bioinformatics/btg412.

692 Peiffer JA., Spor A., Koren O., Jin Z., Tringe SG., Dangl JL., Buckler ES., Ley RE. 2013. Diversity 693 and heritability of the maize rhizosphere microbiome under field conditions. Proceedings 694 of the National Academy of Sciences 110:6548-6553. DOI: 10.1073/pnas.1302837110.

695 Pili NN., França SC., Kyndt T., Makumba BA., Skilton R., Höfte M., Mibey RK., Gheysen G. 696 2015. Analysis of fungal endophytes associated with rice roots from irrigated and upland 697 ecosystems in Kenya. Plant and Soil:1-10. DOI: 10.1007/s11104-015-2590-6.

698

699

700

701

Redman RS., Kim YO., Woodward CJDA., Greer C., Espino L., Doty SL., Rodriguez RJ. 2011. 702 Increased Fitness of Rice Plants to Abiotic Stress Via Habitat Adapted Symbiosis: A 703 Strategy for Mitigating Impacts of Climate Change. PLoS ONE 6. DOI: 10.1371/journal.pone.0014823.

705

Rehman, Sayeed, Mohd Akhtar, Siti Nor Akmar Abdullah 2016. Plant, Soil and Microbes.

706

Rodriguez RJ., White Jr JF., Arnold AE., Redman RS. 2009. Fungal endophytes: diversity and 707 functional roles. New Phytologist 182:314-330. DOI: 10.1111/j.1469-8137.2009.02773.x. 708 Sanders IR., Clapp JP., Wiemken A. 1996. The genetic diversity of arbuscular mycorrhizal fungi 709 in natural ecosystems - a key to understanding the ecology and functioning of the 710 mycorrhizal symbiosis. New Phytologist 133:123-134. DOI: 10.1111/j.14698137.1996.tb04348.x.

712 Santos-Medellín C., Edwards J., Liechty Z., Nguyen B., Sundaresan V. 2017. Drought Stress 713 Results in a Compartment-Specific Restructuring of the Rice Root-Associated Microbiomes. mBio 8:e00764-17. DOI: 10.1128/mBio.00764-17. 
715 Schloss PD., Westcott SL., Ryabin T., Hall JR., Hartmann M., Hollister EB., Lesniewski RA., 716 Oakley BB., Parks DH., Robinson CJ., Sahl JW., Stres B., Thallinger GG., Horn DJV., 717 Weber CF. 2009. Introducing mothur: Open-Source, Platform-Independent, Community718 Supported Software for Describing and Comparing Microbial Communities. Applied and 719 Environmental Microbiology 75:7537-7541. DOI: 10.1128/AEM.01541-09.

720 Seema B. Sharma, Thivakaran A. Gobi 2016. Impact of Drought on Soil and Microbial Diversity 721 722 in Different Agroecosystems of the Semiarid Zones. In: Plant, Soil and Microbes. Springer International Publishing, 149-162.

723 Selosse M-A., Baudoin E., Vandenkoornhuyse P. 2004. Symbiotic microorganisms, a key for 724 ecological success and protection of plants. Comptes Rendus Biologies 327:639-648.

Shakya M., Gottel N., Castro H., Yang ZK., Gunter L., Labbé J., Muchero W., Bonito G., Vilgalys R., Tuskan G., Podar M., Schadt CW. 2013. A Multifactor Analysis of Fungal and Bacterial 729 Community Structure in the Root Microbiome of Mature Populus deltoides Trees. PLoS ONE 8. DOI: 10.1371/journal.pone.0076382.

Silva DD., Kane ME., Beeson RC. 2012. Changes in Root and Shoot Growth and Biomass Partition Resulting from Different Irrigation Intervals for Ligustrum japonicum Thunb. HortScience 47:1634-1640.

Singh L. 2011. Unraveling the role of fungal symbionts in plant abiotic stress tolerance. Plant Signaling \& Behaviour 6:175-191.

734 Smith SE., Read D. 2008. Mycorrhizal Symbiosis (Third Edition). London: Academic Press, 1-9. 735 DOI: 10.1016/B978-012370526-6.50002-7.

736 737 738 739 740

Stamatakis A. 2014. RAxML version 8: a tool for phylogenetic analysis and post-analysis of large phylogenies. Bioinformatics (Oxford, England) 30:1312-1313. DOI: 10.1093/bioinformatics/btu033.

Tchabi A., Coyne D., Hountondji F., Lawouin L., Wiemken A., Oehl F. 2008. Arbuscular mycorrhizal fungal communities in sub-Saharan Savannas of Benin, West Africa, as 
741

742

743 Torrecillas E., Alguacil MM, Roldán A. 2012. Host Preferences of Arbuscular Mycorrhizal Fungi

744

745

746

747

748

749

750

751

752

753

754

755

756

757

758

759

760

761

762

763

764

765

affected by agricultural land use intensity and ecological zone. Mycorrhiza 18:181-195. DOI: $10.1007 / \mathrm{s} 00572-008-0171-8$.

Colonizing Annual Herbaceous Plant Species in Semiarid Mediterranean Prairies. Applied and Environmental Microbiology 78:6180-6186. DOI: 10.1128/AEM.01287-12.

Trenberth KE., Dai A., van der Schrier G., Jones PD., Barichivich J., Briffa KR., Sheffield J. 2014. Global warming and changes in drought. Nature Climate Change 4:17-22. DOI: 10.1038/nclimate2067.

Valladares F., Sanchez-Gomez D., Zavala MA. 2006. Quantitative estimation of phenotypic plasticity: bridging the gap between the evolutionary concept and its ecological applications. Journal of Ecology 94:1103-1116. DOI: 10.1111/j.1365-2745.2006.01176.x.

Vandenkoornhuyse P., Baldauf SL., Leyval C., Straczek J., Young JPW. 2002. Extensive Fungal Diversity in Plant Roots. Science 295:2051-2051. DOI: 10.1126/science.295.5562.2051.

Vandenkoornhuyse P., Quaiser A., Duhamel M., Le Van A., Dufresne A. 2015. The importance of the microbiome of the plant holobiont. New Phytologist 206:1196-1206. DOI: 10.1111/nph.13312.

Vandenkoornhuyse P., Ridgway KP. Watson IJ., Fitter AH., Young JPW. 2003. Co-existing grass species have distinctive arbuscular mycorrhizal communities. Molecular Ecology 12:3085-3095. DOI: 10.1046/j.1365-294X.2003.01967.x.

Verbruggen E., Kiers ET. 2010. Evolutionary ecology of mycorrhizal functional diversity in agricultural systems. Evolutionary Applications 3:547-560. DOI: 10.1111/j.17524571.2010.00145.x.

Vujanovic V., Mavragani D., Hamel C. 2012. Fungal communities associated with durum wheat production system: A characterization by growth stage, plant organ and preceding crop. Crop Protection 37:26-34. DOI: 10.1016/j.cropro.2012.02.006.

Peer] reviewing PDF | (2018:11:32645:1:1:NEW 26 May 2019) 
766 Wagner MR., Lundberg DS, del Rio TG, Tringe SG, Dangl JL., Mitchell-Olds T. 2016. Host

767

768

769

770

771

772

773

774

775

776

777

778

779

780

781

782

783

784

785

786 genotype and age shape the leaf and root microbiomes of a wild perennial plant. Nature Communications 7:12151. DOI: 10.1038/ncomms12151.

Yang T., Ma S., Dai C c. 2014. Drought degree constrains the beneficial effects of a fungal endophyte on Atractylodes lancea. Journal of Applied Microbiology 117:1435-1449. DOI: $10.1111 /$ jam.12615.

Yuan Z., Su Z., Mao L., Peng Y., Yang G., Lin F., Zhang C. 2011. Distinctive endophytic fungal assemblage in stems of wild rice (Oryza granulata) in China with special reference to two species of Muscodor (xylariaceae). The Journal of Microbiology 49:15-23. DOI: $10.1007 / \mathrm{s} 12275-011-0213-3$.

Zhang J., Wang F., Che R., Wang P., Liu H., Ji B., Cui X. 2016. Precipitation shapes communities of arbuscular mycorrhizal fungi in Tibetan alpine steppe. Scientific Reports 6:23488. DOI: $10.1038 /$ srep 23488 .

Zhang T., Yao Y-F. 2015. Endophytic Fungal Communities Associated with Vascular Plants in the High Arctic Zone Are Highly Diverse and Host-Plant Specific. PLoS ONE 10. DOI: 10.1371/journal.pone.0130051.

Zhao K., Tung CW., Eizenga GC., Wright MH., Ali ML., Price AH., Norton GJ., Islam MR., Reynolds A., Mezey J., McClung AM., Bustamante CD., McCouch SR. 2011. Genomewide association mapping reveals a rich genetic architecture of complex traits in Oryza sativa. Nat Commun 2:467. DOI: 10.1038/ncomms 1467. 
Figure 1 (on next page)

Description of the fungal community in the present study.

A total of 447757 sequences were analyzed that belonged to 902 OTUs. Bars represent the logarithmic value of the number of OTUs per taxonomic group. The OTU richness per phylum, subphylum, class and order are shown. 
A

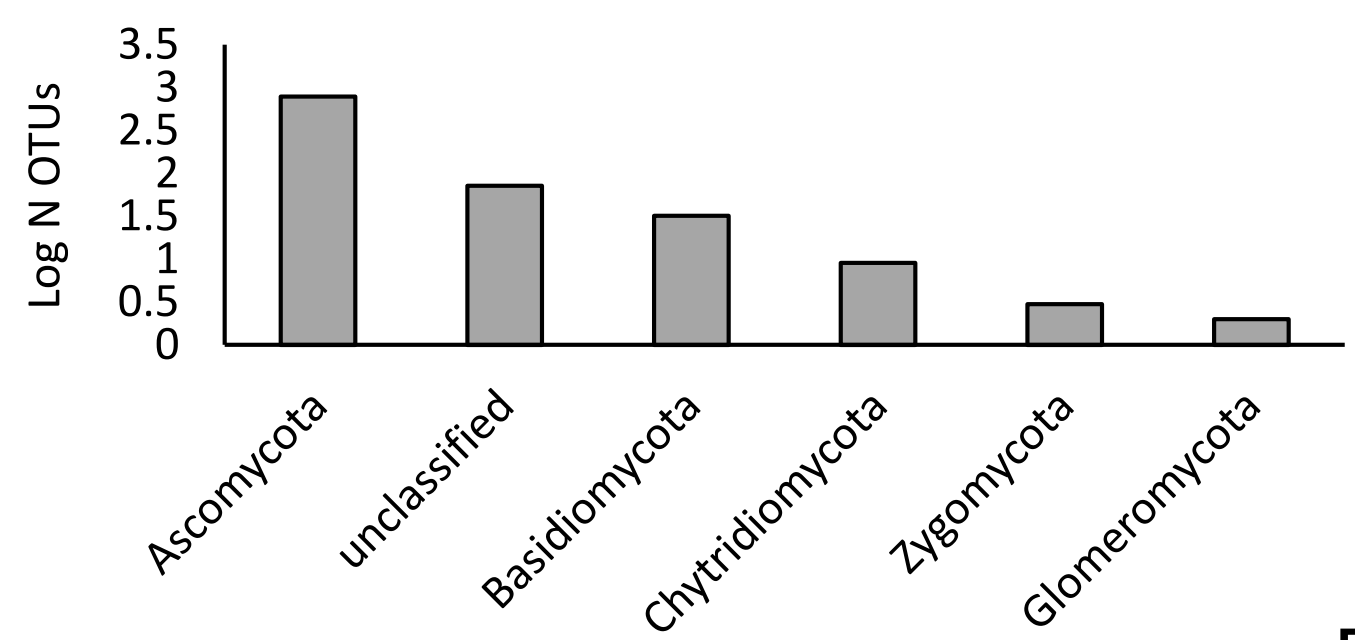

C

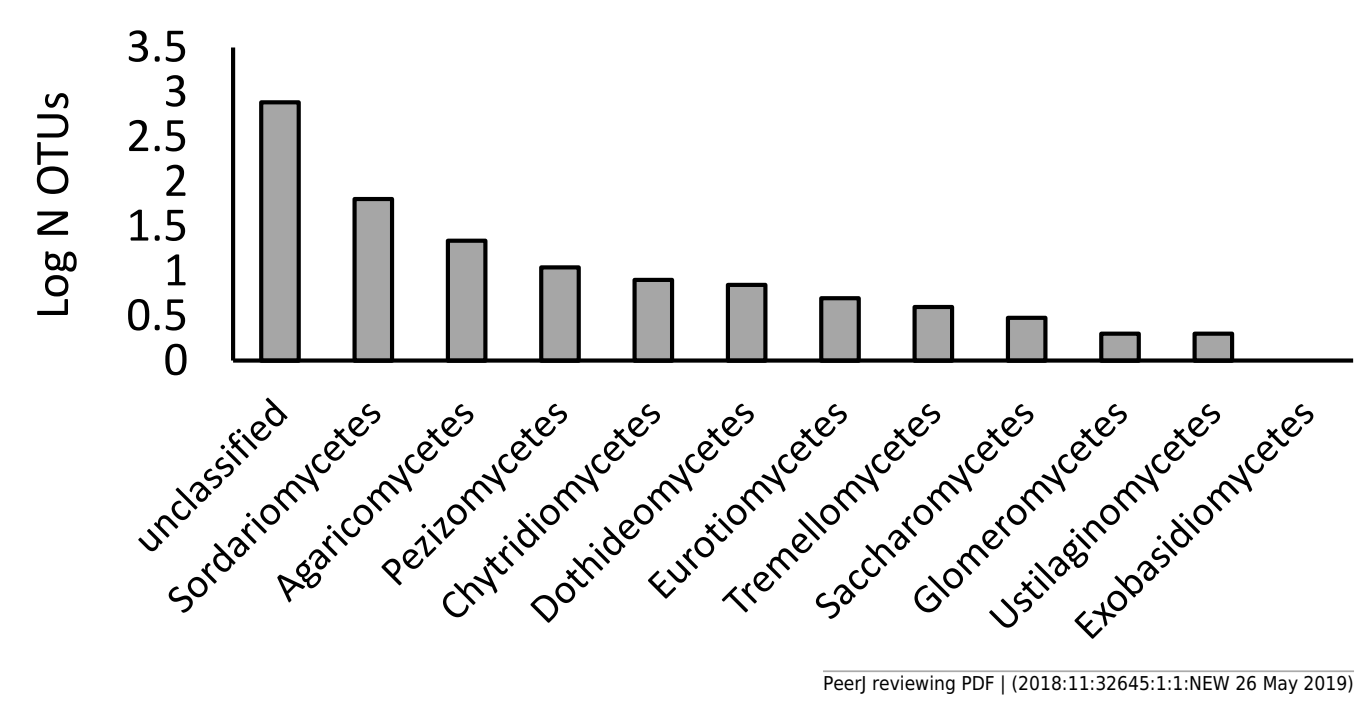

B

Subphylum

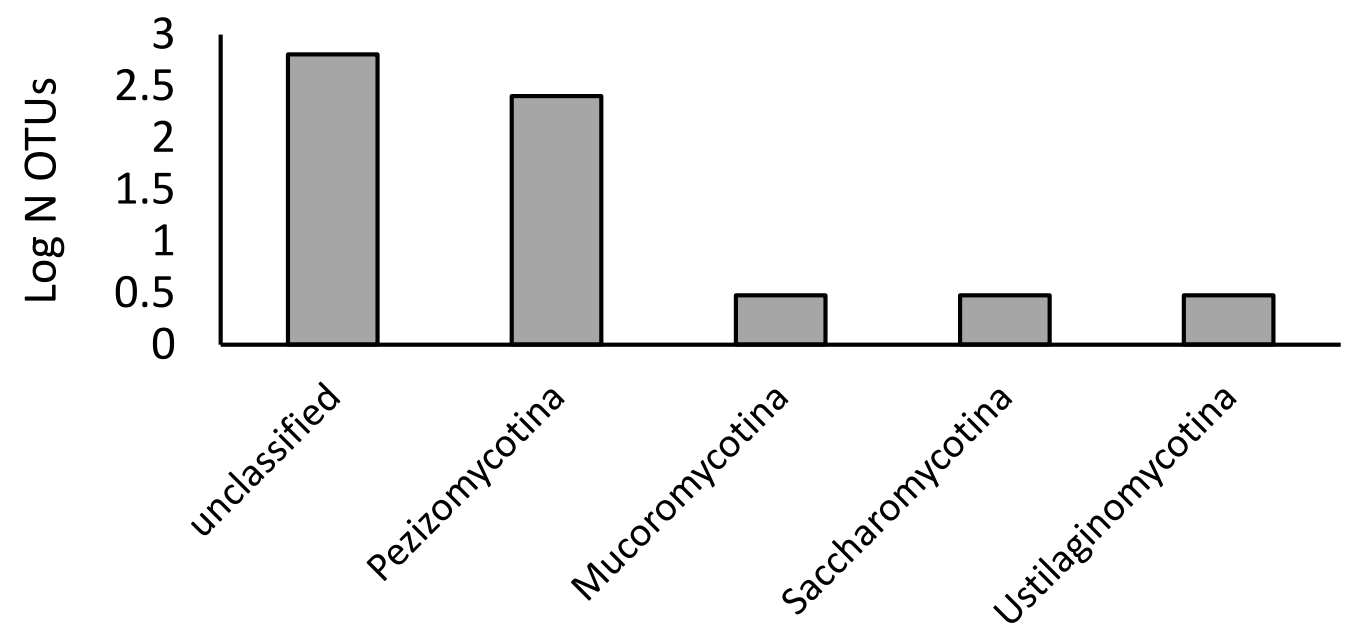

D

Order

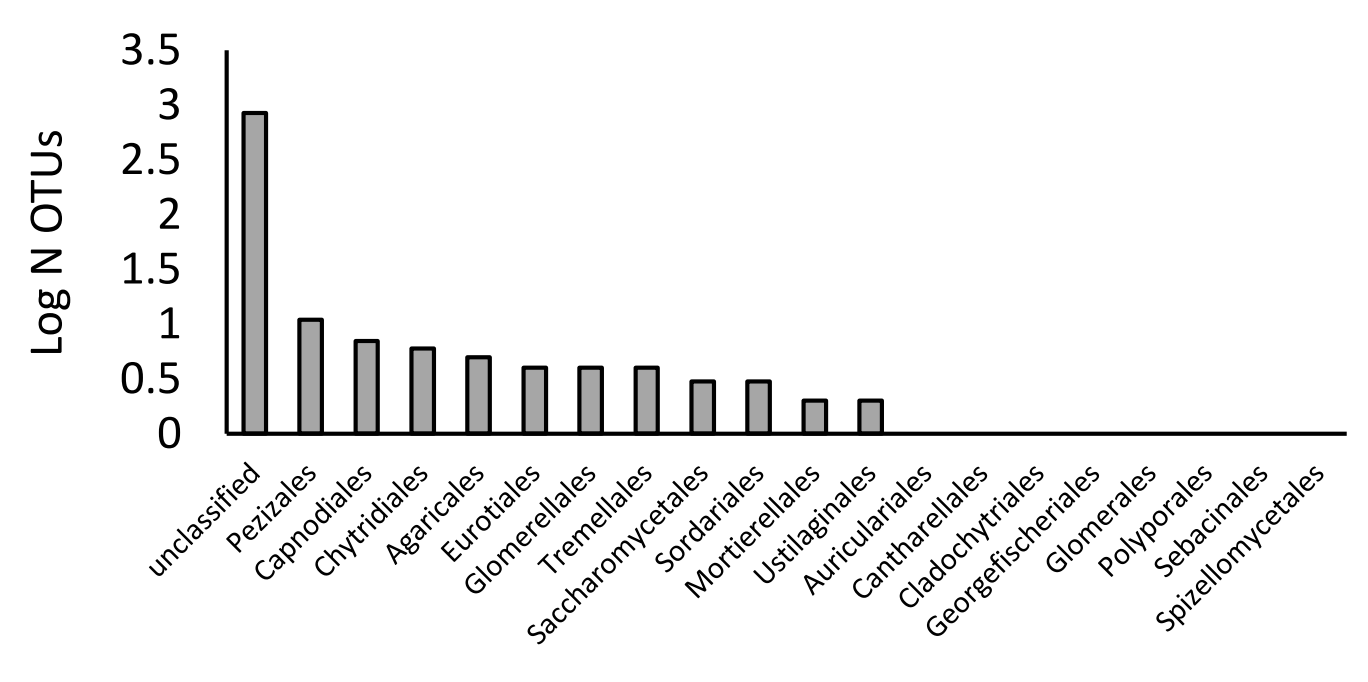


Figure 2 (on next page)

Diversity Shannon index and richness for the different phyla.

(a) Diversity Shannon index and (b) richness for the different phyla, under control (white) and drought (grey) conditions (i.e. $\alpha$-diversity). Results show that OTU richness do not differ much between treatments for all the taxa. On the other hand, diversity is higher under drought for Ascomycota and Basidiomycota, while the unclassified group shows the opposite trend. 


\section{A \\ Pastomycota}

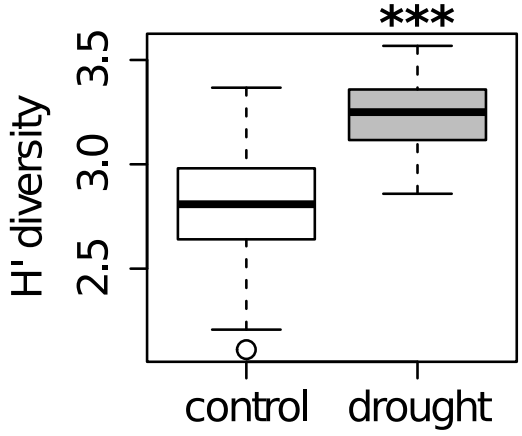

D

Glomeromycota

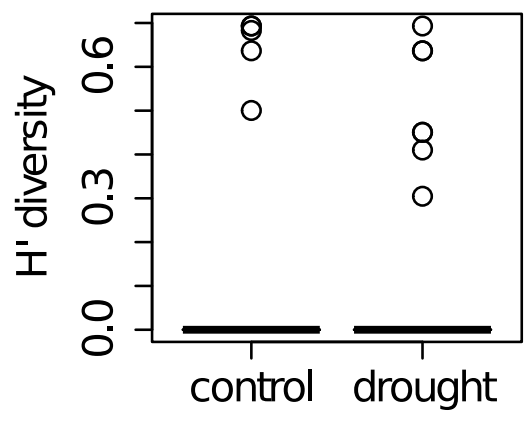

G
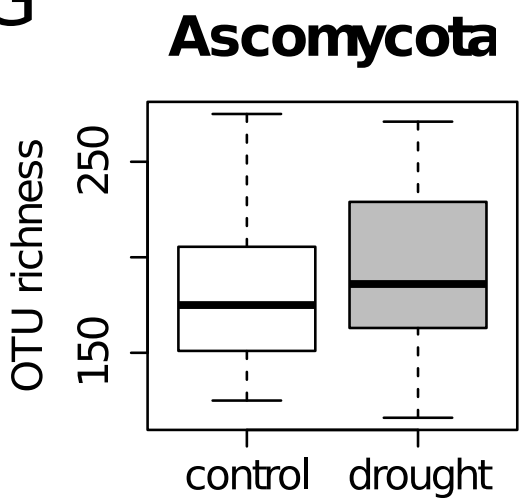

J

Glomeromycota

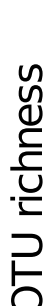

궁

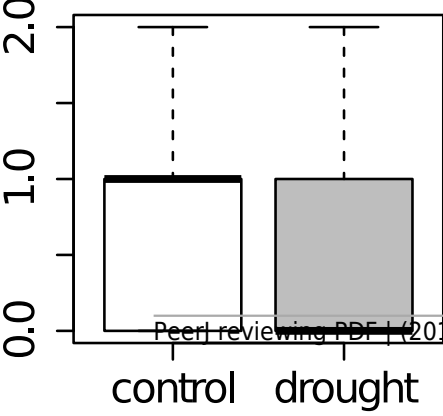

B

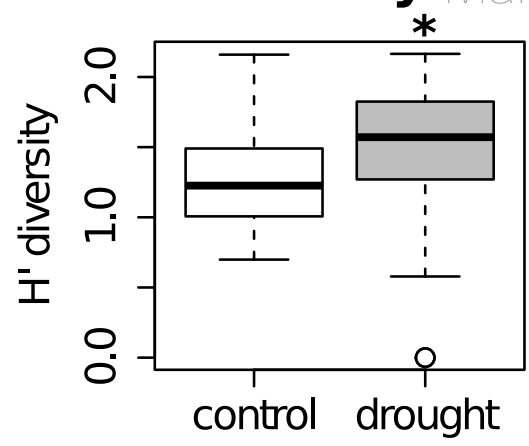

E

F

unclassified

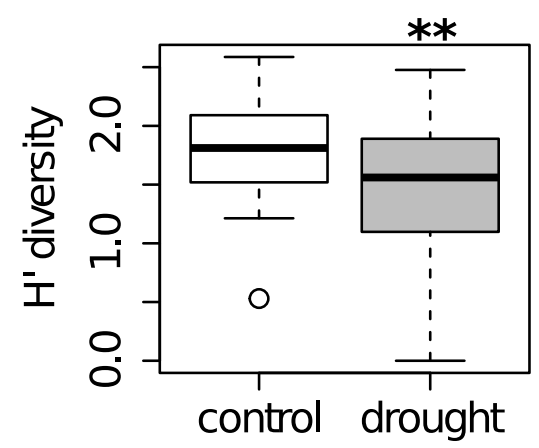

H Basidiomycota

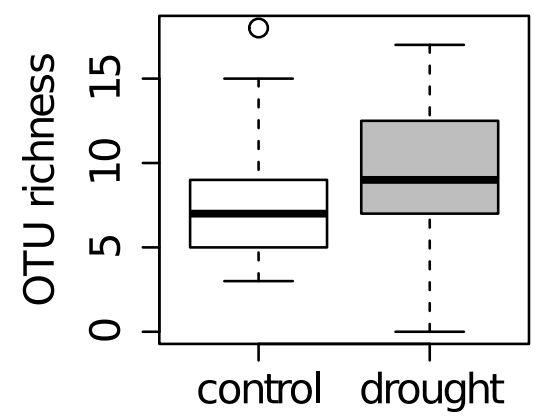

$\mathrm{K}$ unclassified

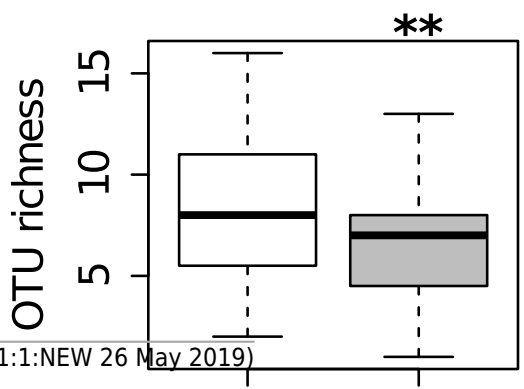

control drought
Zygomycota

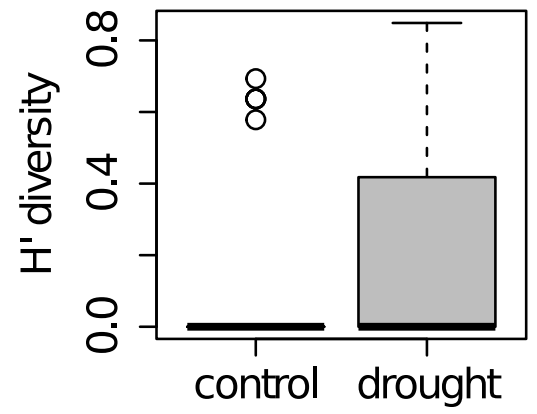

Chytridiomycota

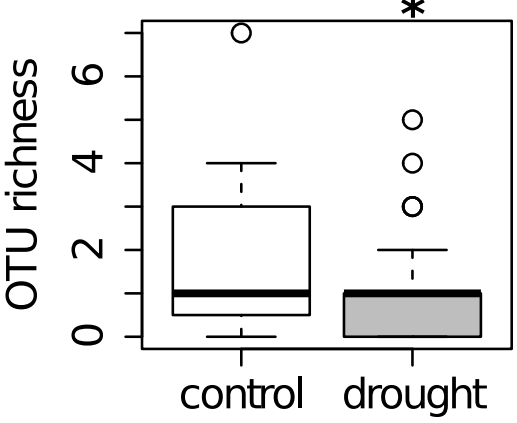

Zygomycota

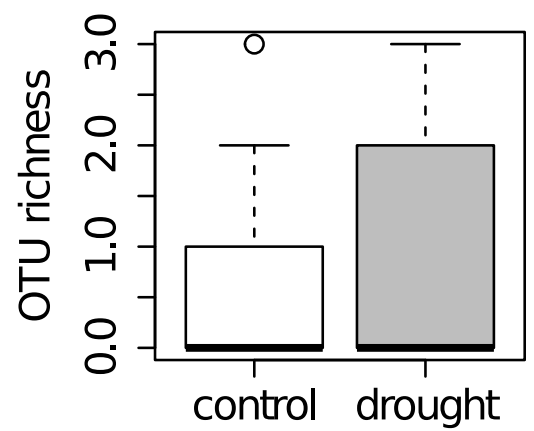


Figure 3 (on next page)

Shannon diversity index for the rice cultivars.

Shannon diversity index for the rice cultivars analyzed, under control (light grey bars) and drought (dark grey bars) (i.e. $\alpha$-diversity). Error bars represent SE. The fungal microbiota Shannon index strongly differs between the treatments (i.e. two-way ANOVA analysis, $\mathrm{P}<0.001)$. 
PeerJ Manuscript to be reviewed

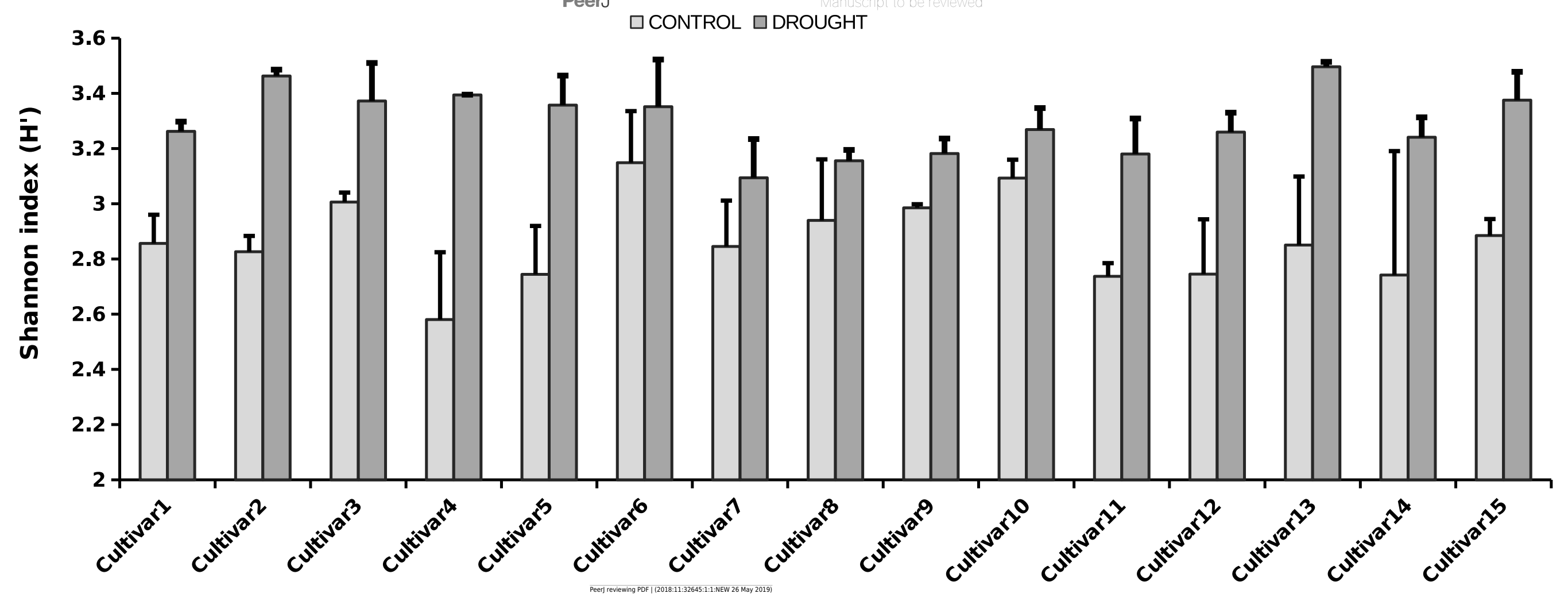


Figure 4 (on next page)

NMDS representing rice root fungal community structure.

A Bray-Curtis dissimilarity distance (i.e. $\beta$-diversity) and a Kulczynski ordination method were used. The statistical analysis (PERMANOVA) showed that the treatments significantly differed in the fungal microbiota composition ( $\mathrm{R} 2=0.37, \mathrm{P}=0.001)$. 
Figure $\mathbf{5}$ (on next page)

Phylogenetic tree.

It represents the 37 OTUs positively correlated with yield under control and drought conditions. The represented OTUs present a correlation value of $\mathrm{R}>0.30$ with a $\mathrm{P}<0.004$. The grey bars provide the OTU occurrence (presence or absence) ratio between treatments: OTU occurrence control - OTU occurrence drought. The occurrence of only 2 of the 37 OTUs remained unchanged between treatments while 22 of the 37 OTUs increased under drought. There is a strong phylogenetic signal between all yield correlated OTUs $(K=6.6 ; P=0.01)$, indicating that yield correlated OTUs are related. 
Tree scale: 0.1

Subdivispons:

Pezizomycotina Unclassified

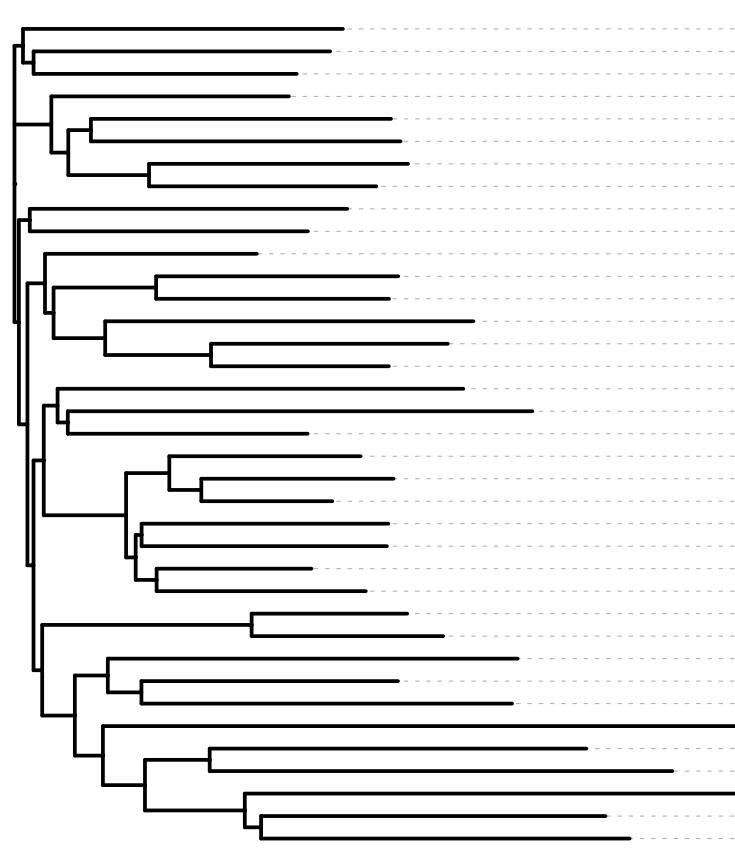

Otu437
Otu681

Otu804

Otu646

Otu193

Otu600

Otu161

Otu548

Otu458

Otu576

Otu544

Otu493

Otu494

Otu571

Otu320

Otu321

Otu184

Otu225

Otu499

Otu467

Otu147
Otu330

Otu551

Otu703

Otu159

Otu507

Otu346

Otu806
Otu099

Otu070

Otu876

Otu001

Otu088

(ture

Otu007

Otu007

Otu197
OTUs correlated withayield in controlreviewed

OTUs correlated with yield in drought $\quad$ OTU occurrence ratio

Drought

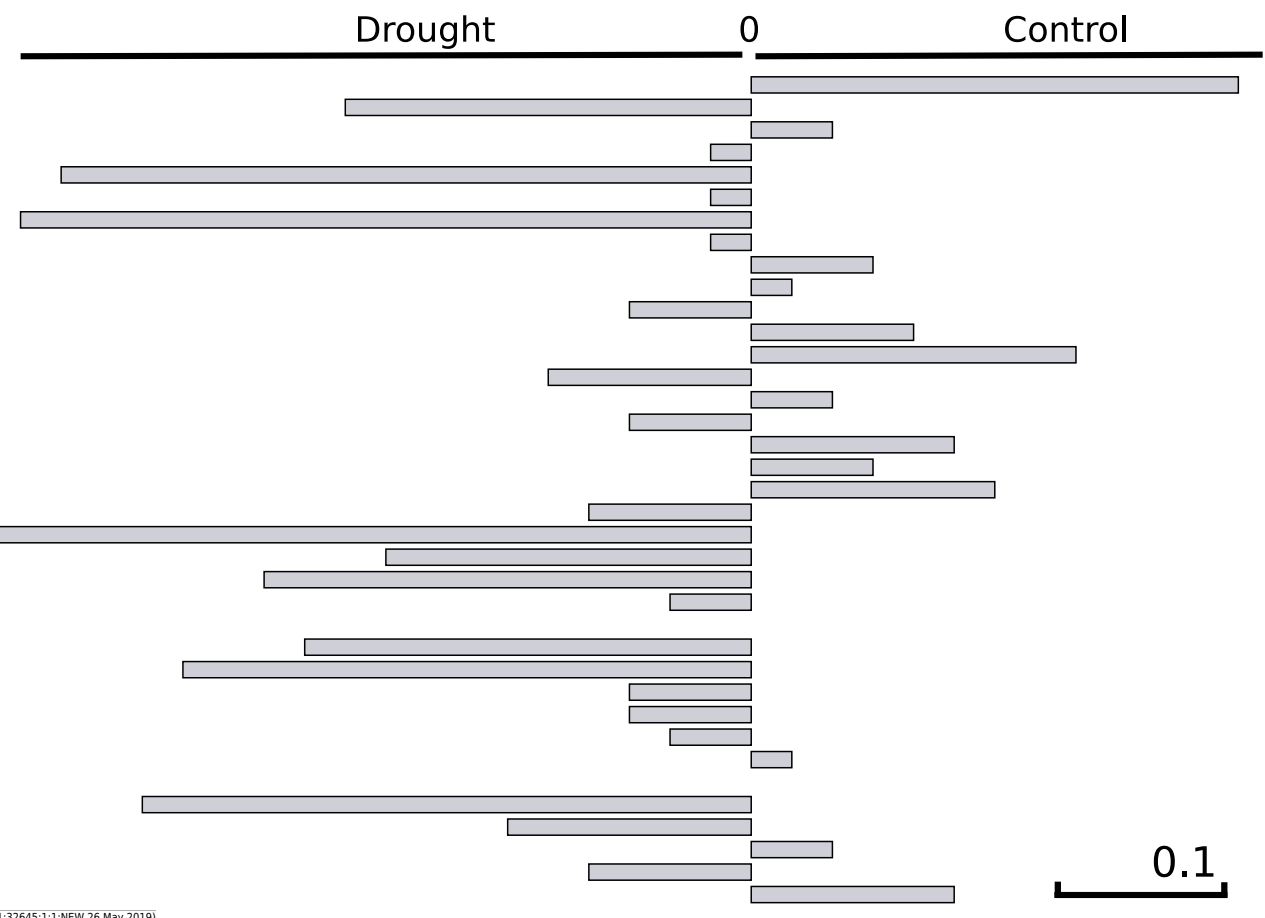

